\title{
AZUFRE ELEMENTAL COMO CORRECTOR DEL pH Y LA FERTILIDAD DE ALGUNOS SUELOS DE LA III Y IV REGIÓN DE CHILE
}

\author{
Elemental sulphur as $\mathrm{pH}$ and soil fertility amendment for some \\ Chileans soils of Regions III and IV
}

\author{
Carlos Sierra B. ${ }^{1}$, Angelus Lancelloti M. ${ }^{2}$, Iván Vidal P. ${ }^{2}$
}

\begin{abstract}
A B S T R A C T
Soils containing calcite are common in Northern Chile, and this condition decreases the availability of nutrients for plants. The effect of the application of elemental sulphur $\left(\mathrm{S}^{\circ}\right)$ was evaluated on soil $\mathrm{pH}$, electrical conductivity and the available micronutrients content in six soils of the III and IV Regions of Chile. This was applied in doses of 500 and 1.000 $\mathrm{mg} \mathrm{S} \mathrm{kg}^{-1}$ and the soils were incubated for periods of 60 and 120 days at $80 \%$ of field capacity and $26^{\circ} \mathrm{C}$. The experimental design was completely randomized with a factorial design and the treatments included soil, doses of elemental sulphur and times of incubation. The soil characteristics having the greatest influence on acidification by sulphur addition were the $\mathrm{CaCO}_{3}$, organic matter and sand contents. A highly significant reduction of soil $\mathrm{pH}$ occured in those soils with a smaller buffering capacity, as a consequence of lower $\mathrm{CaCO}_{3}$ and organic matter contents. Electrical conductivity increased by the application of elemental sulphur as a result of the rise of soluble salts in the soil. When the $\mathrm{pH}$ diminished significantly, the levels of $\mathrm{Fe}, \mathrm{Mn}$ and $\mathrm{Cu}$ micronutrients increased in the soils; Mn was the most strongly influenced by the acidification. This information is useful for the application of amendment programs for the calcareous soils of Northern Chile.
\end{abstract}

Key words: alkaline soils, $\mathrm{pH}$, electrical conductivity, micronutrients.

\section{R E S U M E N}

En la zona norte de Chile existen suelos con carbonatos, situación que influye sobre la disponibilidad de nutrientes para los cultivos. En el presente experimento se evaluó en condiciones de laboratorios, el efecto de la aplicación de azufre elemental $\left(\mathrm{S}^{\circ}\right)$ sobre el $\mathrm{pH}$, conductividad eléctrica y micronutrientes en seis suelos de la III y IV Región, Chile. El azufre se aplicó en dosis de 500 y $1.000 \mathrm{mg} \mathrm{S}^{\circ} \mathrm{kg}^{-1}$ y el suelo se incubó por períodos de 60 y 120 días a $80 \%$ de su capacidad de campo a $25^{\circ} \mathrm{C}$. El experimento se estableció con un diseño de tratamientos completamente al azar con arreglo factorial, donde los factores fueron: suelo, dosis de azufre elemental y tiempo de incubación. Los recipientes en incubación se distribuyeron de acuerdo a un diseño completamente al azar. Las características de los suelos que más influyeron sobre la magnitud del efecto acidificante del azufre elemental fueron los contenidos de $\mathrm{CaCO}_{3}$, materia orgánica y arena. Las reducciones de $\mathrm{pH}$ significativas se presentaron en los suelos con una menor capacidad tampón, como consecuencia del menor contenido de $\mathrm{CaCO}_{3} \mathrm{y}$ materia orgánica. La conductividad eléctrica se incrementó por la aplicación del azufre elemental, debido al aumento de sales solubles en el suelo. Los niveles de los micronutrientes $\mathrm{Fe}, \mathrm{Mn}$ y $\mathrm{Cu}$ se incrementaron en los suelos cuyos $\mathrm{pH}$ disminuyeron significativamente, siendo el Mn el más influenciado por la acidificación. Esta información es de utilidad para establecer programas de aplicación de enmiendas en suelos calcáreos de la zona norte de Chile.

Palabras clave: suelos alcalinos, $\mathrm{pH}$, conductividad eléctrica, micronutrientes.

\footnotetext{
${ }^{1}$ Instituto de Investigaciones Agropecuarias, Centro Regional de Investigación Intihuasi, Colina San Joaquín s/n, Apartado postal 36/B, La Serena, Chile. E-mail: csierra@inia.cl*Autor para correspondencia.

${ }^{2}$ Universidad de Concepción, Facultad de Agronomía, Casilla 537, Chillán, Chile. E-mail: ividal@udec.cl

Recibido: 9 de enero de 2006. Aceptado: 8 de mayo de 2006.
} 


\section{INTRODUCCIÓN}

En el norte de Chile, principalmente desde Copiapó a Ovalle, existe una cantidad importante de suelos alcalinos que presentan un alto $\mathrm{pH}$ y presencia de $\mathrm{CaCO}_{3}$, los cuales afectan la disponibilidad de elementos minerales esenciales para el desarrollo de cultivos. Estos suelos pueden presentar una acumulación de sales solubles y un alto contenido de sodio intercambiable. Muchos de ellos se consideran dentro de la categoría de suelos salinos, por poseer una conductividad eléctrica mayor a $4 \mathrm{dS}$ $\mathrm{m}^{-1} \mathrm{y}$ otros en categoría de suelos sódicos por presentar un valor mayor a $15 \%$ de sodio intercambiable (Brady, 1990; Bohn et al., 1993; Tan, 1993; Salgado, 1996). La concentración de sales reduce la absorción de agua por la planta (Brady, 1990) y el exceso de sodio afecta la estructura, aireación y permeabilidad del suelo, lo que interfiere en el crecimiento de la mayoría de los cultivos. Este problema afecta el cultivo en diverso grado dependiendo de la especie de planta y del grado de alcalinización (Porta et al., 1994).

La reacción del suelo puede influenciar indirectamente el crecimiento de la planta a través de su efecto en la disponibilidad de nutrientes para los cultivos (Tan, 1993). Las deficiencias de micronutrientes tales como hierro $(\mathrm{Fe})$, zinc $(\mathrm{Zn})$, manganeso $(\mathrm{Mn})$ y cobre $(\mathrm{Cu})$ son problemas frecuentes en suelos alcalinos (Brady, 1990; Cifuentes y Lindemann, 1993; Tan, 1993). Por ejemplo, la carencia de hierro en estos suelos es un problema común en la producción de muchos frutales, debido a la inducción de clorosis férrica. Para corregir este tipo de deficiencias nutricionales se han propuesto diferentes métodos para disminuir el $\mathrm{pH}$ de los suelos alcalinos, siendo una alternativa la adición de azufre elemental $\left(\mathrm{S}^{\circ}\right)$ (Fenn et al., 1990; Cifuentes y Lindemann, 1993; Miyamoto, 1998).

El azufre al ser aplicado al suelo está sujeto a oxidación microbiológica, a través de la cual se transforma a sulfato en condiciones aeróbicas, lo que conduce a una disminución del $\mathrm{pH}$ del suelo (Brady, 1990; Deng y Dick, 1990; Miyamoto, 1998).

Los microorganismos que intervienen en el proceso de oxidación del azufre son principalmente del género Thiobacillus, siendo Thiobacillus thiooxidans la especie más importante (Chapman, 1990; Miyamoto, 1998). Sin embargo, otros autores señalan que bacterias heterotróficas y autotróficas facultativas del suelo, pueden ser de mayor relevancia, debido a que se encuentran en mayor cantidad que el género Thiobacillus (Miyamoto, 1998). También, sugieren que el segundo género es importante en la oxidación del azufre sólo a pH ácido, y que las heterotróficas son las principales en suelos neutros a alcalinos (Miyamoto, 1998).

Existen además de los microorganismos del suelo, factores físicos que influyen en la tasa de oxidación del azufre. Los más importantes son: humedad, temperatura, tipo de suelo y tamaño de la partícula de azufre (Deng y Dick, 1990). La temperatura que debe tener el suelo para la oxidación del azufre se encuentra en el rango de 4 a $45^{\circ} \mathrm{C}$, con un rango óptimo entre los 25 y $40^{\circ} \mathrm{C}$ para la mayoría de ellos (Deng y Dick, 1990; Miyamoto, 1998).

Se ha demostrado que con potenciales de agua cercanos a capacidad de campo, la oxidación del azufre procede más rápidamente (Miyamoto, 1998), con un menor contenido de agua, la actividad microbiana se ve limitada y se reduce la accesibilidad de las partículas de azufre, que es altamente hidrofóbico. Por el contrario, con un contenido alto de humedad, la oxidación está limitada posiblemente por una reducción de la aireación del suelo. Por otra parte, la tasa de oxidación del azufre mineral se incrementa con una disminución del diámetro de la partícula, siendo mayor cuando éstas presentan un tamaño menor a $0,14 \mathrm{~mm}$, es decir cuando pasan la malla de 100 mesh. Además, la oxidación del azufre es una reacción exclusivamente superficial y las moléculas del exterior de las partículas están expuestas a una mayor actividad química y biológica. Consecuentemente, la cantidad de sulfato producido por unidad de tiempo es una función total del área superficial del azufre y no de su masa.

Se han realizado estudios del efecto que provocan las sales de los suelos, principalmente de sodio, sobre la oxidación del azufre, y se ha encontrado que una concentración de $\mathrm{NaCl}$ mayor a $9 \%$ reduce su tasa de oxidación, inhibiéndola en 11\% (Miyamoto, 1998). En estos suelos moderadamente sódicos, la tolerancia de los microorganismos oxidantes de azufre a altos niveles de sales libres y sodio intercambiable son un factor importante que influye en su grado de oxidación.

Debido a que no se dispone de información de la oxidación de azufre en los suelos de la III y IV Región, así como su efecto sobre otras características 
del suelo, se propuso la presente investigación con el objetivo de evaluar la factibilidad de la aplicación de azufre elemental como corrector de $\mathrm{pH}$, conductividad eléctrica y disponibilidad de micronutrientes en seis suelos de la III y IV Región.

\section{MATERIALES Y MÉTODOS}

Se colectaron seis muestras superficiales $(0-20 \mathrm{~cm})$ de suelos de diferentes localidades de la III y IV Región del país (Cuadro 1), en base a su contenido calcáreo y su importancia como suelos representativos de la actividad agrícola del norte chico.

Las muestras se secaron al aire y se tamizaron por una malla de $2 \mathrm{~mm}$. Posteriormente, se analizó textura (método del hidrómetro de Bouyoucos), retención de agua a $1 / 3$ y $15 \mathrm{~atm}$ (método de la olla a presión), pH al agua (relación suelo:agua de 1:2,5), materia orgánica (método Walkley y Black), conductividad eléctrica (CE, en relación suelo:agua de 1:5), nitrógeno disponible (extracción de $\mathrm{KCl}$ y después destilación Kjeldahl), fósforo disponible (P Olsen), carbonato de calcio equivalente (tratamiento con ácido clorhídrico), bases intercambiables (extracción con acetato de amonio a pH 7) y micronutrientes hierro $(\mathrm{Fe})$, zinc $(\mathrm{Zn})$, manganeso $(\mathrm{Mn})$ y cobre $(\mathrm{Cu})$ extracción con ácido dietilentriaminopentaacético (DTPA). Los análisis se realizaron en el Laboratorio del Centro Regional de Investigación (CRI) La Platina, acreditado por la Sociedad Chilena de la Ciencia del Suelo.

Se pesaron submuestras equivalentes a $400 \mathrm{~g}$ de suelo seco, a las que se les añadió azufre elemental $\left(\mathrm{S}^{\circ}\right)$ puro, ventilado, con un tamaño de partícula menor de 325 mesh $(0,045 \mathrm{~mm})$, en dosis de $500 \mathrm{y}$ $1.000 \mathrm{mg} \mathrm{kg}^{-1}$ de suelo, las cuales se depositaron en bolsas de polietileno y se mezclaron completamen- te para obtener una distribución homogénea en el suelo. Posteriormente se adicionó agua destilada suficiente para mantener el suelo a un $80 \%$ de su capacidad de campo. Las muestras se incubaron a $25^{\circ} \mathrm{C}$ en una estufa (Heraeus Instruments D-63450, Hanau, Alemania) por períodos de 60 y 120 días. En forma similar se incubaron muestras como testigos sin adición de azufre elemental, las cuales se pesaron periódicamente para evaluar pérdidas en el contenido de humedad, y así mantenerlas al mismo contenido de agua original.

Al término de cada período de incubación, las submuestras se secaron al aire para posteriormente determinar el $\mathrm{pH}$ al agua ( $\mathrm{Fe}, \mathrm{Mn}, \mathrm{Zn}$ y $\mathrm{Cu}$ ), lo que se analizó en el Laboratorio de Fertilidad de Suelos del Centro Regional de Investigación (CRI) La Platina, de acuerdo a la metodología descrita por Sadzawka (1990).

El experimento se estableció con tres repeticiones por tratamiento y con un diseño completamente al azar con arreglo factorial, donde los factores fueron suelo (6) (Cuadro 1), dosis de $S^{\circ}(3)$ : 0; 500; y $1.000 \mathrm{mg} \mathrm{kg}^{-1}$ y tiempo de incubación (2). Los resultados se analizaron para conocer su varianza y prueba de rango múltiple para comparación de medias de Tukey al 5\%, y así detectar diferencias entre tratamientos. Además, se realizó análisis de correlación lineal para examinar las relaciones entre algunas propiedades de cada suelo y la diferencia de los $\mathrm{pH}$ determinados.

\section{RESULTADOS Y DISCUSIÓN}

\section{Características de los suelos y efecto de los tra- tamientos}

Algunas características físicas y químicas de cada suelo evaluado se presentan en el Cuadro 2. El sue-

Cuadro 1. Nombre y ubicación de las series de seis suelos de la III y IV Región de Chile.

Table 1. Names and location of six soils of Regions III and IV, Chile.

\begin{tabular}{llllcc}
\hline Serie & Orden & Comuna & Localidad & Latitud & Longitud \\
\hline Toledo & Entisols & Copiapó & Bodega & $27^{\circ} 22^{\prime}$ & $70^{\circ} 23^{\prime}$ \\
La Compañía & Aridisols & Vallenar & La Compañía & $28^{\circ} 34^{\prime}$ & $70^{\circ} 49^{\prime}$ \\
Xeres & Aridisols & Coquimbo & Pan de Azúcar & $29^{\circ} 59^{\prime}$ & $71^{\circ} 17^{\prime}$ \\
Reciente $^{1}$ & Entisols & Río Hurtado & Pichasca & $30^{\circ} 26^{\prime}$ & $70^{\circ} 51^{\prime}$ \\
Reciente $^{1}$ & Entisols & Punitaqui & El Higueral & $30^{\circ} 49^{\prime}$ & $71^{\circ} 16^{\prime}$ \\
Reciente $^{1}$ & Entisols & Punitaqui & Pueblo Viejo & $30^{\circ} 51^{\prime}$ & $71^{\circ} 15^{\prime}$ \\
\hline
\end{tabular}

${ }^{1}$ Estos suelos fueron referidos en el texto con el nombre de la localidad. 


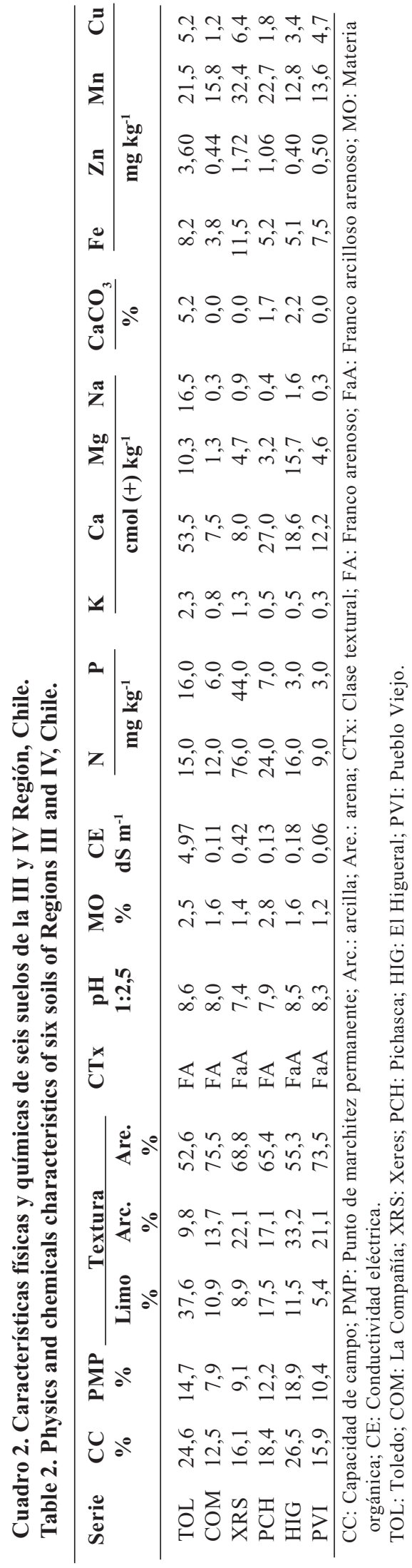

lo Toledo posee mayor $\mathrm{pH}$, más alto contenido de $\mathrm{CaCO}_{3}$, y CE de $4,97 \mathrm{dS} \mathrm{m}^{-1}$, por lo tanto clasifica como suelo salino. Por otro lado, los suelos restantes presentan una CE selectivamente baja, y no alcanzan un valor sobre el cual se considerarían salinos. Los suelos La Compañía y Pueblo Viejo presentan mayor contenido de arena 75,5 y $73,5 \%$, respectivamente; en tanto, el Higueral posee más arcilla, con un $33,2 \%$, y el segundo con más alto $\mathrm{pH}$. Aunque todos los suelos poseen un alto contenido de materia orgánica, el de mayor valor es el suelo Pichasca seguido por Toledo. Con respecto al contenido de micronutrientes disponibles en el suelo, todos se encuentran en concentraciones normales, con la excepción de los suelos La Compañía y El Higueral que presentan déficit de $\mathrm{Zn}$.

En el Cuadro 3 se presentan los valores de los cuadrados medios y su grado de significancia. En él se observan diferencias altamente significativas $(\mathrm{P} \leq 0,01)$ en el factor suelo para todas las variables estudiadas. Las características físicas y químicas de ellos explicarían estas diferencias (Cuadro 2). De igual manera, la dosis de aplicación de $\mathrm{S}^{\circ}$ influyó en forma altamente significativa en todas las variables evaluadas. En tanto, el tiempo de incubación sólo afectó el $\mathrm{pH}$, disponibilidad de Fe y Zn. Las otras variables, como CE y disponibilidad de $\mathrm{Mn}$ y $\mathrm{Cu}$, no se vieron influenciadas por ese tratamiento aplicado.

La interacción suelo $\times$ dosis de $\mathrm{S}^{\circ}$ fue altamente significativa en todas las variables, excepto en la $\mathrm{CE}$ del suelo. Esto sugiere que el contenido salino del suelo al aplicar $\mathrm{S}^{\circ}$ no varió de manera distinta entre los distintos suelos estudiados. Por otra parte, el $\mathrm{pH}$ y la disponibilidad de Fe se afectaron en forma altamente significativa por la interacción de los tres factores estudiados.

\section{Efecto del azufre elemental $\left(\mathrm{S}^{\circ}\right)$ sobre la reac- ción del suelo $(\mathrm{pH})$}

Los valores promedio de $\mathrm{pH}$ para la interacción de los factores suelo $\times$ dosis de $\mathrm{S}^{\circ} \times$ tiempo de incubación se presentan en el Cuadro 4 . Se infiere que en todos los suelos hubo una disminución del $\mathrm{pH}$ por efecto de la aplicación de $\mathrm{S}^{\circ}$, como consecuencia de la transformación a sulfato por oxidación microbiológica. La magnitud de esta caída fue incrementada con el aumento de la dosis de $\mathrm{S}^{\circ}$. Por otro lado, en la generalidad de los suelos, los tratamientos sin aplicación de $\mathrm{S}^{\circ}$ sufrieron un decrecimiento 
Cuadro 3. Valores de los cuadrados medios para las distintas evaluaciones realizadas en seis suelos de la III y IV Región, Chile.

Table 3. Average mean square values for the different evaluations obtained in six soils of Regions III and IV, Chile.

\begin{tabular}{lcccccc}
\hline \multicolumn{1}{c}{ Factor } & pH & $\begin{array}{c}\text { Conductividad } \\
\text { eléctrica }\end{array}$ & Hierro & Zinc & Manganeso & Cobre \\
\hline Suelo & $11,94^{* *}$ & $60,24^{* *}$ & $656,15^{* *}$ & $21,99^{* *}$ & $14.836,2^{* *}$ & $79,53^{* *}$ \\
Dosis de S ${ }^{\circ}$ & $14,92^{* *}$ & $1,49^{* *}$ & $322,86^{* *}$ & $0,20^{* *}$ & $35.727,1^{* *}$ & 7,98 \\
Tiempo de incubación & $1,42^{* *}$ & n.s. & $53,20^{* *}$ & $0,66^{* *}$ & n.s. & n.s. \\
Suelo x dosis de S & $1,62^{* *}$ & n.s. & $102,79^{* *}$ & $0,06^{* *}$ & $8.019,1 * *$ & 1,48 \\
Suelo x tiempo de incubación & $0,36 * *$ & n.s. & $31,47^{* *}$ & n.s. & n.s. & n.s. \\
Dosis de S ${ }^{\circ}$ x tiempo incubación & $0,42^{* *}$ & n.s. & $54,16^{* *}$ & n.s. & n.s. & n.s. \\
Suelo x dosis S ${ }^{\circ}$ x tiempo incubación & $0,08^{* *}$ & n.s. & $18,03^{* *}$ & n.s. & n.s. & n.s. \\
Error & 0,0229 & 0,09 & 4,69 & 0,02 & 241,37 & 0,55 \\
CV $(\%)$ & 21,06 & 24,94 & 25,03 & 10,61 & 20,53 & 19,62 \\
\hline
\end{tabular}

** Significativos a la probabilidad de 0,01 .

n.s.: valores no significativos; ${ }^{\circ} \mathrm{S}$ : azufre elemental; CV: coeficiente de variación.

leve de pH después de la incubación (Cuadro 2), debido a una ligera acidificación natural, atribuible a la mineralización del nitrógeno orgánico existente en el suelo, producto de la liberación de $\mathrm{CO}_{2}$.

El análisis de varianza muestra diferencias altamente significativas $(\mathrm{P} \leq 0,01)$ entre todos los factores y sus interacciones (Cuadro 3). Los suelos Toledo y Pichasca presentaron menos variación del $\mathrm{pH}$ por efecto de la aplicación de $\mathrm{S}^{\circ}$. Aunque existió una cierta tendencia al decrecimiento, éste no fue significativo (Cuadro 4).

Por el contrario, la acidificación en el suelo La Compañía fue mucho más efectiva, tanto con el aumento de la dosis de $\mathrm{S}^{\circ}$ como con el tiempo de incubación. Del mismo modo, el suelo Xeres presentó una alta respuesta a la aplicación de $\mathrm{S}^{\circ}$, especialmente con el mayor tiempo de incubación. Ambos suelos no presentan calcita, esto explica el claro efecto del $\mathrm{S}^{\circ}$ sobre la acidificación. Por otro lado, el suelo El Higueral tuvo un comportamiento intermedio, en donde existieron diferencias sólo entre los tratamientos sin dosis de $\mathrm{S}^{\circ}$ con respecto a los tratados con aplicación de $\mathrm{S}^{\circ}$. Se detectó la presencia de $2,2 \%$ de carbonatos, que explica el menor efecto de la adición de $\mathrm{S}^{\circ}$ sobre la acidificación del suelo.

La mayor respuesta a la aplicación de $\mathrm{S}^{\circ}$ se presentó en el suelo Pueblo Viejo, que de un pH inicial de 7,8 bajó a 4,5 en el segundo período de incubación. La oxidación del $\mathrm{S}^{\circ}$ se manifestó claramente a los 60 días de incubación del suelo, donde se ve una diferencia altamente significativa entre los tratamientos testigos respecto a los tratados con la dosis más alta (Cuadro 4).

Cuadro 4. Valores promedio de $\mathrm{pH}$ en agua para la interacción suelo por dosis de azufre elemental $\left(\mathrm{S}^{\circ}\right)$ y por tiempo de incubación.

Table 4. pH average value for the interaction between soil and elemental sulphur $\left(\mathrm{S}^{\circ}\right)$ rate and incubation time.

\begin{tabular}{|c|c|c|c|c|}
\hline \multirow[t]{2}{*}{$\begin{array}{l}\text { Días de } \\
\text { incubación }\end{array}$} & \multicolumn{4}{|c|}{$\begin{array}{c}\text { pH en agua } \\
\text { Dosis de } S^{\circ}\left(\mathrm{mg} \mathrm{kg}^{-1}\right)\end{array}$} \\
\hline & 0 & 500 & 1.000 & Promedio \\
\hline \multicolumn{5}{|l|}{ Toledo } \\
\hline 60 días & $8,38 \mathrm{a}$ & $8,20 \mathrm{a}$ & $8,09 \mathrm{a}$ & \\
\hline 120 días & $8,31 \mathrm{a}$ & $8,17 \mathrm{a}$ & $8,03 \mathrm{a}$ & 8,20 \\
\hline \multicolumn{5}{|l|}{ La Compañía } \\
\hline 60 días & $7,71 \mathrm{a}$ & $6,67 \mathrm{~b}$ & $6,49 \mathrm{~b}$ & \\
\hline 120 días & $7,59 \mathrm{a}$ & $6,38 \mathrm{~b}$ & $5,62 \mathrm{c}$ & 6,74 \\
\hline \multicolumn{5}{|l|}{ Xeres } \\
\hline 60 días & $7,51 \mathrm{a}$ & $6,52 \mathrm{~b}$ & $5,75 \mathrm{c}$ & \\
\hline 120 días & $7,32 \mathrm{a}$ & $5,89 \mathrm{c}$ & $4,92 \mathrm{~d}$ & 6,32 \\
\hline \multicolumn{5}{|l|}{ Pichasca } \\
\hline 60 días & $7,45 \mathrm{a}$ & $7,40 \mathrm{a}$ & $7,31 \mathrm{a}$ & \\
\hline 120 días & $7,66 \mathrm{a}$ & $7,50 \mathrm{a}$ & $7,30 \mathrm{a}$ & 7,44 \\
\hline \multicolumn{5}{|l|}{ El Higueral } \\
\hline 60 días & $8,39 \mathrm{a}$ & $7,91 \mathrm{~b}$ & $7,63 \mathrm{~b}$ & \\
\hline 120 días & $8,39 \mathrm{a}$ & $7,92 \mathrm{~b}$ & $7,71 \mathrm{~b}$ & 7,99 \\
\hline \multicolumn{5}{|l|}{ Pueblo Viejo } \\
\hline 60 días & $7,76 \mathrm{a}$ & $6,58 \mathrm{~b}$ & $5,53 \mathrm{c}$ & \\
\hline 120 días & $7,79 \mathrm{a}$ & $6,15 \mathrm{~b}$ & $4,50 \mathrm{~d}$ & 6,39 \\
\hline
\end{tabular}

Letras minúsculas diferentes en sentido vertical y horizontal, dentro de cada suelo indican diferencias entre la interacción, según la prueba de Tukey $(\mathrm{P} \leq 0,05)$. 
Las diferentes variaciones sufridas por el $\mathrm{pH}$ de los suelos se explicarían por algunas características físicas y químicas que éstos poseen. Para esto se realizó una correlación lineal entre las diferencias de pH y algunas de estas características. La escasa variación del $\mathrm{pH}$ al aplicar azufre de los suelos Toledo y Pichasca se debe al contenido de $\mathrm{CaCO}_{3}$ presente, avalado por el coeficiente de correlación, el cual fue significativo e inverso $\left(r=-0,70^{*}\right)$. Al respecto, está altamente demostrado que el $\mathrm{CaCO}_{3}$ reacciona con el $\mathrm{H}_{2} \mathrm{SO}_{4}$ producido y lo neutraliza, permitiendo al suelo tener una capacidad reguladora o tampón (Lindemann et al., 1991; Cifuentes y Lindemann, 1993; Miyamoto, 1998).

La relación entre la magnitud del cambio de $\mathrm{pH}$ y el contenido de materia orgánica fue significativa e inversa $\left(r=-0,68^{*}\right)$, es decir, los suelos con mayor contenido de materia orgánica presentaron una mayor resistencia al cambio por efecto de la adición de $\mathrm{S}^{\circ}$.

Así también, otro factor que tuvo incidencia fue el contenido de arena de los suelos, presentándose una respuesta más efectiva a la aplicación de $\mathrm{S}^{\circ}$ en los suelos con mayor contenido de arena, como fue el caso de los suelos Pueblo Viejo y La Compañía. La correlación entre estas dos variables fue significativa $\left(r=0,67^{*}\right)$. Es muy probable que esta relación con la arena se explique por fragmentos de carbonato de calcio del tamaño de ésta que no fueron eliminados antes de hacer el análisis de textura.

De acuerdo a lo señalado anteriormente, las variables de mayor incidencia en la magnitud del efecto acidificante en el suelo por la adición de $\mathrm{S}^{\circ}$, correspondieron al contenido de $\mathrm{CaCO}_{3}$, materia orgánica y porcentaje de arena, los cuales explican en mayor proporción el cambio de $\mathrm{pH}$ por la aplicación de $\mathrm{S}^{\circ}$.

\section{Efecto del azufre elemental $\left(\mathrm{S}^{\circ}\right)$ sobre la conduc- tividad eléctrica del suelo (CE)}

El efecto de la dosis de $\mathrm{S}^{\circ}$ sobre la CE de los suelos estudiados se presenta en el Cuadro 5. La dosis de $\mathrm{S}^{\circ}$ influyó en forma altamente significativa sobre la CE (Cuadro 3), y su efecto fue diferente de acuerdo al tipo de suelo.

La serie Toledo presenta los mayores valores de CE y no se afectó por la aplicación de $\mathrm{S}^{\circ}$. La diferencia con respecto a los demás suelos radica principalmente por su condición de suelo salino.

El contenido de sales de un suelo es comúnmente reflejado en la CE del extracto de saturación. El incremento de la $\mathrm{CE}$ por efecto de la aplicación de $\mathrm{S}^{\circ}$ se debería a la reacción del $\mathrm{H}_{2} \mathrm{SO}_{4}$ con el $\mathrm{CaCO}_{3}$, que provee de iones $\mathrm{Ca}$ a la solución del suelo, generándose $\mathrm{CaSO}_{4}$, con el consiguiente incremento del contenido de aniones y cationes, como lo señalan algunos investigadores (Lindemann et al., 1991; Miyamoto, 1998). Esto se confirma con una correlación significativa entre la CE y la diferencia de $\mathrm{pH}$ de los suelos evaluados $\left(\mathrm{r}=0,67^{*}\right)$.

Efecto del azufre elemental $\left(\mathrm{S}^{\circ}\right)$ sobre los micronutrientes hierro, zinc, manganeso y cobre

En el Cuadro 6 se presentan los valores promedio de Fe disponible, al considerar la interacción de los

Cuadro 5. Valores promedio de la conductividad eléctrica de los suelos para los factores suelo y dosis de azufre elemental $\left(\mathrm{S}^{\circ}\right)$.

Table 5. Average value for the electrical conductivity of the soils as related to the rate of $S^{\circ}$ applied.

\begin{tabular}{|c|c|c|c|c|}
\hline \multirow[t]{2}{*}{ Suelo } & \multicolumn{4}{|c|}{$\begin{array}{c}\text { Conductividad eléctrica }\left(\mathrm{dS} \mathrm{m}^{-1}\right) \\
\text { Dosis } \mathrm{S}^{\circ}\left(\mathrm{mg} \mathrm{kg}^{-1}\right)\end{array}$} \\
\hline & $\mathbf{0}$ & 500 & 1.000 & Promedio \\
\hline Toledo & 4,75 & 4,74 & 4,79 & $4,76 \mathrm{~A}$ \\
\hline La Compañía & 0,12 & 0,43 & 0,57 & $0,37 \mathrm{~B}$ \\
\hline Xeres & 0,45 & 0,68 & 0,78 & $0,64 \mathrm{~B}$ \\
\hline Pichasca & 0,27 & 0,46 & 0,59 & $0,44 \mathrm{~B}$ \\
\hline El Higueral & 0,24 & 0,45 & 0,61 & $0,43 \mathrm{~B}$ \\
\hline Pueblo Viejo & 0,17 & 0,34 & 0,59 & $0,37 \mathrm{~B}$ \\
\hline Dosis de $\mathrm{S}^{\circ}$ & $0,99 \mathrm{c}$ & $1,18 \mathrm{~b}$ & $1,40 \mathrm{a}$ & 1,19 \\
\hline
\end{tabular}

Letras mayúsculas diferentes en sentido vertical indican diferencias en el factor suelo, según la prueba de Tukey $(\mathrm{P} \leq 0,05)$.

Letras minúsculas diferentes en sentido horizontal, dentro de cada suelo indican diferencias en el sector dosis de azufre elemental, según la prueba de Tukey $(\mathrm{P} \leq 0,05)$. 
Cuadro 6. Valores promedio de hierro disponible para la interacción suelo $\times$ dosis de azufre elemental $\times$ tiempo de incubación.

Table 6. Average values of available iron in relation with the interaction between soil $x$ elemental sulphur rate $x$ incubation time.

\begin{tabular}{|c|c|c|c|c|}
\hline \multirow[t]{2}{*}{ Suelo } & \multicolumn{4}{|c|}{$\begin{array}{c}\mathrm{pH} \text { al agua } \\
\text { Dosis de azufre elemental }\left(\mathrm{mg} \mathrm{kg}^{-1}\right)\end{array}$} \\
\hline & $\mathbf{0}$ & 500 & 1.000 & Promedio \\
\hline \multicolumn{5}{|l|}{ Toledo } \\
\hline 60 días & $7,93 \mathrm{a}$ & $8,17 \mathrm{a}$ & $8,93 \mathrm{a}$ & \\
\hline 120 días & $7,33 \mathrm{a}$ & $7,10 \mathrm{a}$ & $7,00 \mathrm{a}$ & 7,74 \\
\hline \multicolumn{5}{|l|}{ La Compañía } \\
\hline 60 días & $3,40 \mathrm{a}$ & $4,13 \mathrm{a}$ & $4,50 \mathrm{a}$ & \\
\hline 120 días & $2,83 \mathrm{a}$ & $4,63 \mathrm{a}$ & $7,33 \mathrm{a}$ & 4,47 \\
\hline \multicolumn{5}{|l|}{ Xeres } \\
\hline 60 días & $11,73 \mathrm{c}$ & $16,00 \mathrm{c}$ & $22,67 \mathrm{~b}$ & \\
\hline 120 días & $10,97 \mathrm{c}$ & $20,17 \mathrm{bc}$ & $34,53 \mathrm{a}$ & 19,35 \\
\hline \multicolumn{5}{|l|}{ Pichasca } \\
\hline 60 días & $4,67 \mathrm{a}$ & $4,63 \mathrm{a}$ & $4,47 \mathrm{a}$ & \\
\hline 120 días & $4,00 \mathrm{a}$ & $3,93 \mathrm{a}$ & $3,93 \mathrm{a}$ & 4,27 \\
\hline \multicolumn{5}{|l|}{ El Higueral } \\
\hline 60 días & $4,20 \mathrm{a}$ & $4,23 \mathrm{a}$ & $4,10 \mathrm{a}$ & \\
\hline 120 días & $4,10 \mathrm{a}$ & $4,20 \mathrm{a}$ & $3,87 \mathrm{a}$ & 4,12 \\
\hline \multicolumn{5}{|l|}{ Pueblo Viejo } \\
\hline 60 días & $6,40 \mathrm{c}$ & $8,33 \mathrm{bc}$ & $14,57 \mathrm{~b}$ & \\
\hline 120 días & $5,40 \mathrm{c}$ & $9,63 \mathrm{bc}$ & $26,97 \mathrm{a}$ & 11,88 \\
\hline
\end{tabular}

Letras minúsculas diferentes en sentido vertical y horizontal, dentro de cada suelo indican diferencias significativas entre la interacción, según la prueba de Tukey $(\mathrm{P} \leq 0,05)$.

factores: suelo, tiempo de incubación y dosis de $\mathrm{S}^{\circ}$. En los suelos Xeres y Pueblo Viejo se observó un mayor impacto de la adición de $\mathrm{S}^{\circ}$ sobre la disponibilidad de Fe, duplicándose los valores originales con la mayor dosis de $\mathrm{S}^{\circ}$ y el tiempo de incubación de 120 días. Esto se explica por la disminución del pH del suelo, a los 120 días de incubación la dosis alta de azufre bajó el pH a menos de 5,0 (Cuadro 4). Este efecto está demostrado por algunos autores (Katyal y Randhawa, 1986), quienes indican que la actividad del Fe se incrementa con el aumento de la acidez de la solución del suelo. Otro factor que puede generar una liberación de $\mathrm{Fe}$ es la reducción del medio, producto de una saturación prolongada del espacio poroso del suelo. Sin embargo, en las condiciones del presente experimento el suelo se mantuvo bajo condiciones de capacidad de campo, aunque es probable que microsectores de la muestras, especialmente la parte inferior de éstas, pueden haber estado sometidas a saturación parcial. Por otra parte, en aquellos suelos con mayor capacidad reguladora del $\mathrm{pH}$ por efecto del contenido de calcita, no hubo efecto significativo de la aplicación de $\mathrm{S}^{\circ}$ sobre la disponibilidad del $\mathrm{Fe}$.
Lo anteriormente señalado se confirma con el coeficiente de correlación, el cual presenta significancia entre el Fe disponible y la magnitud de reducción del $\mathrm{pH}$ obtenido en los suelos evaluados $\left(\mathrm{r}=0,84^{*}\right)$.

El efecto de la dosis de $\mathrm{S}^{\circ}$ sobre el $\mathrm{Zn}$ disponible en los suelos evaluados se presenta en el Cuadro 7, observándose que los suelos Toledo y Xeres fueron los únicos que presentaron un incremento significativo de este elemento con la aplicación de $\mathrm{S}^{\circ}$. Por otro lado, en el resto de los suelos, si bien la disponibilidad del $\mathrm{Zn}$ aumentó ligeramente, este cambio no fue significativo. Cabe señalar que no se presentó correlación significativa entre el pH y los niveles de $\mathrm{Zn}$.

Los valores promedio de Mn disponible en los suelos para las diferentes dosis de $\mathrm{S}^{\circ}$ se muestran en el Cuadro 7. La disponibilidad de Mn se incrementó con la reducción del $\mathrm{pH}$, y este aumento es mayor cuando se alcanzan $\mathrm{pH}$ inferiores a 6,5. En efecto, los suelos La Compañía, Xeres y Pueblo Viejo, triplicaron los contenidos de Mn disponible cuando alcanzaron $\mathrm{pH}$ próximos a 6,5 por la adición de la 


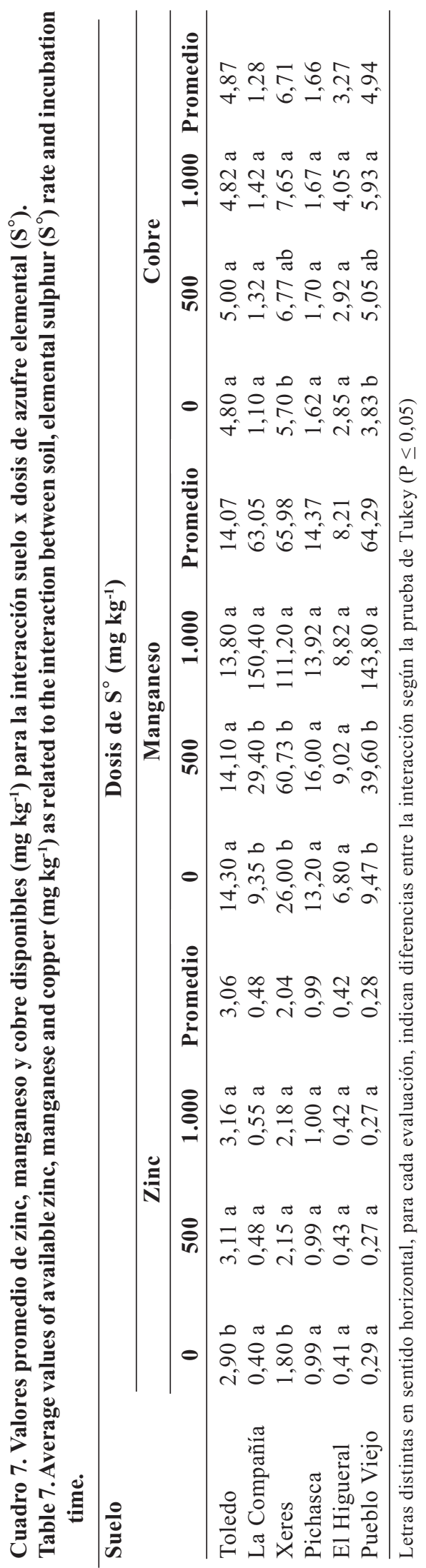

dosis de $500 \mathrm{mg} \mathrm{S}^{\circ} \mathrm{kg}^{-1}$. Con $1.000 \mathrm{mg} \mathrm{S}^{\circ} \mathrm{kg}^{-1}$, la disponibilidad de manganeso se incrementó más aún, alcanzando entre 4 y 15 veces el contenido de los tratamientos testigos. Estos resultados confirman que el Mn es el micronutriente que posee una mayor respuesta a la acidificación, demostrando que su solubilidad y disponibilidad es muy dependiente del $\mathrm{pH}$ del suelo; sin embargo, igualmente la disponibilidad del manganeso es incrementada por condiciones de reducción del suelo.

El coeficiente de correlación indica un efecto positivo entre las disminuciones sufridas por el $\mathrm{pH}$ de los suelos y la disponibilidad de $\mathrm{Mn}\left(\mathrm{r}=0,83^{*}\right)$, lo que confirma lo anteriormente señalado.

La disminución de $\mathrm{pH}$ incrementa el Mn en la solución del suelo a valores que pueden llegar a ser tóxicos para la mayoría de los cultivos, principalmente con valores de $\mathrm{pH}$ menores a 5,0. Por otra parte, se reconoce que el secado induce un aumento del Mn extractable, por lo cual estos valores se podrían deber a que estos suelos fueron analizados después de un secado, influenciando un incremento del nutriente.

El efecto de la dosis de $\mathrm{S}^{\circ}$ sobre el $\mathrm{Cu}$ disponible analizado se muestra en el Cuadro 7. Los suelos Xeres, El Higueral y Pueblo Viejo presentaron los mayores aumentos entre los tratamientos con aplicación de $\mathrm{S}^{\circ}$.

La solubilidad del $\mathrm{Cu}$ es dependiente del $\mathrm{pH}$ del suelo, pero normalmente no aumenta en forma apreciable hasta que el $\mathrm{pH}$ cae por debajo de un valor de 5,0, como se demuestra en los suelos Xeres y Pueblo Viejo, donde el $\mathrm{Cu}$ disponible tuvo un aumento significativo (Cuadro 7). Los suelos restantes no incrementaron en forma apreciable la disponibilidad del nutriente. Lo anteriormente señalado se comprueba por la correlación lineal de la disponibilidad del $\mathrm{Cu}$ en los suelos con respecto a la magnitud del decrecimiento del $\mathrm{pH}\left(\mathrm{r}=0,73^{*}\right)$.

La información reportada en el presente trabajo permite señalar que la respuesta del suelo a la aplicación de $\mathrm{S}^{\circ}$ dependerá del contenido de calcita y materia orgánica. En suelos calcáreos, la disponibilidad de micronutrientes no aumentará de manera importante al aplicar $\mathrm{S}^{\circ}$, mientras que en suelos no calcáreos ésta puede incrementarse de manera notable, como el caso del manganeso. Por lo tanto, 
la dosificación de $\mathrm{S}^{\circ}$ como enmienda acidificante debe ser previamente bien definida, analizando no sólo el $\mathrm{pH}$, sino también la presencia de carbonatos y materia orgánica del suelo.

\section{CONCLUSIONES}

Como consecuencia de la aplicación de azufre elemental al suelo, las variables de mayor incidencia sobre la magnitud en reducción del $\mathrm{pH}$ sobre el suelo correspondieron al contenido de $\mathrm{CaCO}_{3}$, materia orgánica y porcentaje de arena. Las dos primeras variables aportan al suelo una mayor capacidad tampón.

La conductividad eléctrica de los suelos tuvo un incremento significativo por efecto de la incorpo- ración de azufre elemental, como consecuencia de una mayor solubilidad de las sales presentes. El efecto fue significativo entre suelos, radicado principalmente en el tipo de suelo.

Aquellos suelos que bajaron en forma significativa su $\mathrm{pH}$ por el efecto acidificante del azufre elemental fueron los que más incrementaron los niveles de micronutrientes disponibles; excepto el zinc, cuyos valores aumentaron independientemente de la acidificación de los suelos.

El manganeso fue el micronutriente más influenciado por los cambios de $\mathrm{pH}$, triplicando sus valores cuando se alcanzó un pH inferior a 6,5.

\section{LITERATURA CITADA}

Bohn, H., B.L. McNeal, y G. O'Connor. 1993. Suelos afectados por sales. p. 259-287. In Grupo Noriegan (ed.) Química del suelo. Ed. Limusa, México, DF.

Brady, N.C. 1990. The nature and properties of soils. $10^{\mathrm{a}}$ ed. 590 p. Prentice-Hall, New York, USA.

Cifuentes, F.R., and W. Lindemann. 1993. Organic matter stimulation of elemental sulfur oxidation in a calcareous soils. Soil Sci. Soc. Am. J. 57:727-731.

Chapman, S.J. 1990. Thiobacillus populations in some agricultural soils. Soil Biol. Biochem. 22:479-482.

Deng, S., and R.P. Dick. 1990. Sulfur oxidation and Rhodanese activity in soils. Soil Sci. 150:552-560.

Fenn, L.B., H.L. Malstrom, T. Riley, and G.L. Horst. 1990. Acidification of calcareous soils improves zinc absorption of pecan trees. J. Am. Soc. Hortic. Sci. 115:741-744.

Katyal, J.C., y N.S. Randhawa. 1986. Micronutrientes. Boletín FAO Fertilizantes y Nutrición Animal. p. 164. Organización de las Naciones Unidas para la Agricultura y la Alimentación, Roma, Italia.

Lindemann, W., J. Aburto, W.M. Haffner, and A.A. Bono. 1991. Effect of sulfur source on sulfur oxidation. Soil Sci. Soc. Am. J. 55:85-90.
Miyamoto, S. 1998. Use of acids and acidulants on Alkali soils and water. p. 217-255. In A. Wallace and R.E. Terry (eds.) Handbook of soil conditioners substances that enhance the physical properties of soil. Part III. Mineral soil conditioners. Marcel Dekker, New York, USA.

Porta, J., M. López-Acevedo, y C. Roquero. 1994. Edafología para la agricultura y el medio ambiente. 807 p. Ed. Mundi Prensa, Madrid, España.

Sadzawka, A. 1990. Métodos de análisis de suelo. Serie La Platina $\mathrm{N}^{\circ} 16.130 \mathrm{p}$. Instituto de Investigaciones Agropecuarias, Estación Experimental La Platina, Santiago, Chile.

Salgado, L. 1996. Conceptos generales sobre salinidad. Boletín de Extensión $\mathrm{N}^{\circ}$ 41. p. 1-43. Universidad de Concepción, Chillán, Chile.

Tan, K.H. 1993. Principles of soil chemistry. $2^{\text {a }}$ ed. 210 p. Marcel Dekker, New York, USA. 